Publ. Mat. 63 (2019), 125-154

DOI: 10.5565 /PUBLMAT6311904

\title{
SULLIVAN MINIMAL MODELS OF OPERAD ALGEBRAS
}

\author{
JoAna Cirici And Agustí Roig
}

\begin{abstract}
We prove the existence of Sullivan minimal models of operad algebras for a quite wide family of operads in the category of complexes of vector spaces over a field of characteristic zero. Our construction is an adaptation of Sullivan's original step by step construction to the setting of operad algebras. The family of operads that we consider includes all operads concentrated in degree 0 as well as their minimal models. In particular, this gives Sullivan minimal models for algebras over Com, Ass, and $\mathcal{L} i e$, as well as over their minimal models $\mathcal{C o m}_{\infty}, \mathcal{A} s s_{\infty}$, and $\mathcal{L i e}_{\infty}$. Other interesting operads, such as the operad Ger encoding Gerstenhaber algebras, also fit in our study.
\end{abstract}

2010 Mathematics Subject Classification: 18D50, 55P62.

Key words: Minimal models, rational homotopy, operad algebras.

\section{Contents}

1. Introduction 126

2. Preliminaries 129

3. Basic homotopy theory of operad algebras 131

4. Sullivan minimal models 135

5. Uniqueness of the minimal model 144

6. Algebras over variable operads 146

7. Chain operad algebras and one example 149

$\begin{array}{ll}\text { References } & 152\end{array}$

Cirici would like to acknowledge financial support from the German Research Foundation (SPP-1786) and partial support from the Spanish Ministry of Economy and Competitiveness (MTM2016-76453-C2-2-P). Roig is partially supported by projects MTM2012-38122-C03-01/FEDER and 2014 SGR 634. 


\section{Introduction}

The classical construction of Sullivan minimal models of commutative differential graded algebras over a field $\mathbf{k}$ of characteristic zero is done step by step by a process of "attaching cells", called KS-extensions (from Koszul-Sullivan) or Hirsch extensions. The data of these KS-extensions is encoded in a graded vector space together with a linear differential, whereas the multiplication of the algebra comes for free, thanks to the notion of free algebra. With this in mind, it is natural to ask whether the cell attachment construction can be extrapolated to a more general context. An obvious candidate is the category of $P$-algebras, where $P$ is an operad in the category of complexes of $\mathbf{k}$-vector spaces.

While $P$-algebras can behave very badly, in the sense that operations with negative degrees can undo the work of previous steps in a cell attachment procedure, many interesting operads given in nature (i.e. geometry, topology, and physics) behave badly, but in a somewhat tame way that we precise here:

Let $P$ be an operad in cochain complexes of $\mathbf{k}$-vector spaces. We will always assume that $P$ is connected $(P(1)=\mathbf{k})$ and that it is either reduced $(P(0)=0)$ or unitary $(P(0)=\mathbf{k})$. Let $r \geq 0$ be an integer. We say that $P$ is $r$-tame if for all $n \geq 2$, we have that

$$
P(n)^{q}=0 \text { for all } q \leq(1-n)(1+r) .
$$

Note that $r$-tame implies $(r+1)$-tame. Examples of 0 -tame operads are: Ass, Com, and Lie, every operad concentrated in degree 0 and the operads $\mathcal{A} s s_{\infty}, \mathcal{C o m}_{\infty}$, and $\mathcal{L} i e_{\infty}$. More generally, minimal models of reduced $r$-tame operads are $r$-tame. An example of 1-tame operad is $\mathrm{G} e r$, the one encoding Gerstenhaber algebras.

In the category of $P$-algebras, there is a notion of free $P$-algebra generated by a graded $\mathbf{k}$-vector space. From this notion, we define $K S$-extensions of free $P$-algebras analogously to the rational homotopy setting of Com-algebras. We say that a $P$-algebra $\mathcal{M}$ is a Sullivan minimal $P$-algebra if it is the colimit of a sequence of $\mathrm{KS}$-extensions starting from $P(0)$, ordered by non-decreasing positive degrees. A Sullivan minimal model of a $P$-algebra $A$ is a Sullivan minimal $P$-algebra $\mathcal{M}$, together with a morphism $f: \mathcal{M} \rightarrow A$ of $P$-algebras whose underlying map of cochain complexes induces an isomorphism in cohomology; i.e., a quasiisomorphism of $P$-algebras. As a warning, let us remark that here and elsewhere along the paper, $f$ and all algebra morphisms are morphisms in the strict sense, not $\infty$-morphisms.

As in the rational homotopy setting, we require cohomological connectedness for our algebras. A $P$-algebra $A$ is called 0 -connected if 
$H^{i}(A)=0$ for all $i<0$ and the unit map $\eta: P(0) \rightarrow A$ induces an isomorphism $P(0) \cong H^{0}(A)$. Let $r \geq 0$. Then $A$ is called $r$-connected if, in addition, we have that $H^{1}(A)=\cdots=H^{r}(A)=0$. We prove:

Theorem 4.6. Let $P$ be an $r$-tame operad. Then every $r$-connected $P$-algebra $A$ has a Sullivan minimal model $f: \mathcal{M} \rightarrow A$ with $\mathcal{M}^{0}=$ $P(0)$ and $\mathcal{M}^{i}=0$ for all $i<r$ with $i \neq 0$. Furthermore, if $A$ is $(r+$ 1)-connected and $H^{*}(A)$ is of finite type, then $\mathcal{M}$ is of finite type.

Note that in the particular case $P=C o m$ we recover Sullivan's theorem of minimal models for commutative differential graded algebras over $\mathbf{k}$. We also obtain Sullivan minimal models for 0 -connected $P$-algebras, when $P$ is one of the operads $\mathcal{A} s s, \mathcal{L} i e, \mathcal{C o m}_{\infty}, \mathcal{A} s s_{\infty}$, or $\mathcal{L} i e_{\infty}$ among others. Furthermore, the above result gives Sullivan minimal models for 1-connected Ger-algebras. All these minimal models are unique:

Theorem 5.3. Let $P$ be an $r$-tame operad and $A$ an $r$-connected $P$-algebra. Let $f: \mathcal{M} \rightarrow A$ and $f^{\prime}: \mathcal{M}^{\prime} \rightarrow A$ be two Sullivan minimal models of $A$. Then there is an isomorphism $g: \mathcal{M} \rightarrow \mathcal{M}^{\prime}$, unique up to homotopy, such that $f^{\prime} g \simeq f$.

Remarks 1.1. A few remarks are in order:

(1) Relation with existing Sullivan minimal models. Sullivan's classical construction of minimal models for commutative differential graded algebras has been adapted to several other algebraic settings. Examples are Quillen's models of differential graded Lie algebras [Qui], the models for chain differential graded (Lie) algebras of Baues-Lemaire $[\mathbf{B L}]$ and Neisendorfer [Nei], the theory of Leibniz algebras of [Liv2] and, more closely related to our approach, the minimal models of chain $P$-algebras, where $P$ is a Koszul operad concentrated in degree 0 , developed by Livernet in her $\mathrm{PhD}$ Thesis [Liv1]. As we show in Section 7, our results are equally valid for cochain and chain algebras, after minor modifications are taken into account. In particular, our work generalizes all of the above mentioned studies. Furthermore, the results of this paper make precise some of the ideas contained in [Sul2], where Sullivan defines triangular $P$-algebras as free $P$-algebras with a partial ordering on their generators and sketches a theory of triangular resolutions.

(2) Koszul duality theory. For Koszul quadratic $P$-algebras, there is a theory of quasi-free resolutions which give minimal models in some situations (see $[\mathbf{L V}]$, [Mil1], $[$ Mil2]). While there is a certain overlap of algebras for which both Koszul duality theory and our Sullivan algorithm for algebras over tame operads apply, let 
us mention some notable differences. First, to know whether an operad is Koszul or not, can prove to be very difficult (see [MSS, Remark 3.98]). The theory developed in this paper doesn't require operads to be Koszul, not even quadratic. In particular, there is no restriction on the height of the relations among their generators. In contrast, we do impose some restrictions on the arity-degree range of the elements of the operad, but this condition is straightforward to verify. Second, while Koszul duality theory applies to quadratic algebras satisfying certain conditions, our algorithm applies to all sufficiently connected $P$-algebras, once the operad $P$ is proven to be tame. Furthermore, we produce minimal models for both unitary, $P(0)=\mathbf{k}$, and non-unitary, $P(0)=0$, algebras, while Koszul duality theory applies only to the latter case. Lastly, let us mention that in Koszul duality theory, minimal models are constructed via the cobar resolution of the associated coalgebra, while, in this paper, we give "step by step" minimal models, following Sullivan's classical approach. This may be useful, for instance, to compute partial minimal models up to a certain degree and extract homotopical information.

(3) Kadeishvili's models. There are many results in the literature about "minimal models" for operad algebras in the $\infty$-sense. Prominently, Kadeishvili [Kad] defined minimal models of $A_{\infty}$-algebras as $A_{\infty}$-algebras with trivial differential. Similarly, there is the homotopy transfer theorem for $P_{\infty}$-algebras (see $[\mathbf{L V}]$ ) and the theory of minimal models for operad algebras developed in $[\mathbf{C L}]$. As it is well-known, minimal models à la Kadeishvili do not correspond to minimal models à la Sullivan, the main differences being that, for the first ones, morphisms are $\infty$-morphisms and minimality is a vanishing condition on the differential, while for the later, morphisms are strict and minimality involves freeness and a certain behavior of the (not-necessarily trivial) differential. However, a characterizing property is shared by the two approaches: every quasi-isomorphism between minimal algebras is an isomorphism.

(4) Minimal models of operads. Every reduced operad $P$ in the category of complexes of $\mathbf{k}$-vector spaces such that $H(P)(1)=\mathbf{k}$ has a minimal model (defined as a free operad whose differential is decomposable). Here we study minimal models of the algebras, and not of the operads themselves. However, there is a relation between the two problems that we address in this paper. The idea is that one can consider the category of algebras above all operads as a fibred category. We show that minimal objects in this category 
are given by those objects that are both minimal on the fiber and the base. This provides a global invariance of our minimal models.

We explain the contents of the paper. In Section 2 we collect wellknown results on operads and operad algebras. In Section 3 we develop the basic homotopy theory of operad algebras. In Section 4 we introduce $r$-tame operads and prove the existence of minimal models for algebras over these operads. We also show that the minimal model of every $r$-tame operad is $r$-tame, and give some examples. Section 5 deals with the uniqueness of our minimal models. In Section 6 we study the fibred category of algebras over all operads and give global minimal models in this case. Lastly, in Section 7 we explain the case of chain operad algebras (with homological degree) and compute an example of $\mathcal{G}_{\infty} r_{\infty}$ minimal model.

\section{Preliminaries}

In this first section, we recall some main constructions for operads and operad algebras in the category of cochain complexes of vector spaces over a field of characteristic 0 and fix notation. For preliminaries on operads, we refer to $[\mathbf{M S S}],[\mathbf{L V}],[\mathbf{F r e}]$, and $[\mathbf{K M}]$. We refer to $[\mathbf{G r M o}$, [FHT], and the original paper of Sullivan [Sul1] for a review of rational homotopy theory.

Throughout this paper, let $\mathbf{k}$ denote a field of characteristic 0 .

2.1. Operads in cochain complexes. We will consider unital symmetric operads in the category of unbounded cochain complexes of vector spaces over $\mathbf{k}$. Denote by $\mathbf{O p}$ the category of such operads.

Given an operad $P$ in $\mathbf{O p}$ we will denote by

$$
\gamma_{l ; m_{1}, \ldots, m_{l}}^{P}: P(l) \otimes P\left(m_{1}\right) \otimes \cdots \otimes P\left(m_{l}\right) \longrightarrow P\left(m_{1}+\cdots+m_{l}\right)
$$

its structure morphisms and by $\eta: \mathbf{k} \rightarrow P(1)$ its unit. These morphisms satisfy equivariance, associativity, and unit axioms (see [MSS, Definition I.4]). Alternatively, we can use the equivalent data of partial composition operations

$$
\mathrm{o}_{i}: P(m) \otimes P(n) \longrightarrow P(m+n-1) .
$$

An operad $P$ is called unitary if $P(0)=\mathbf{k}$ is concentrated in degree 0 . It is called reduced if $P(0)=0$. We will say that $P$ is connected if $P(1)=\mathbf{k}$ is concentrated in degree 0 . In this paper we will always consider connected operads that are either unitary or reduced.

2.2. Operad algebras. Let $P \in \mathbf{O p}$ be an operad. Denote by $\mathbf{A} \lg _{P}$ the category of $P$-algebras. For a $P$-algebra $A$, we will denote by $\theta_{A}(l): P(l) \otimes_{\Sigma_{l}} A^{\otimes l} \rightarrow A$ its structure morphisms. These are subject to natural associativity and unit constraints (see $[\mathbf{K M}]$ ). 
Since every $P$-algebra has an underlying cochain complex, we have a notion of quasi-isomorphism in $\mathbf{A l g}_{P}$ given by those morphisms of $P$-algebras whose underlying morphism of cochain complexes induces an isomorphism in cohomology.

We next recall some constructions in the category of $P$-algebras that will be used in the sequel.

2.3. Functorial properties (c.f. $[\mathbf{L V}, 5.2 .14])$. Every morphism of operads $F: P \rightarrow Q$ induces a reciprocal image or restriction of scalars functor $F^{*}: \mathbf{A l g}_{Q} \rightarrow \mathbf{A} \lg _{P}$ defined on objects $B \in \mathbf{A} \mathbf{l g}_{Q}$ by the compositions $\theta_{F^{*} B}(l)=\theta_{B}(l) \circ\left(F(l) \otimes \mathrm{id}_{B}^{\otimes l}\right): P(l) \otimes_{\Sigma_{l}} B^{\otimes l} \rightarrow B$. Note that $F^{*}$ preserves quasi-isomorphisms and surjective morphisms, since the underlying complexes remain unchanged.

2.4. Tensor product. Let $P, Q \in \mathbf{O p}$ be two operads. Their pointwise tensor product is the operad $P \otimes Q$ whose arity $l$ is the cochain complex $P(l) \otimes Q(l)$. Given a $P$-algebra $A$ and a $Q$-algebra $B$, their tensor product as cochain complexes $A \otimes B$ has a natural structure of $(P \otimes Q)$-algebra. The operad Com being the unit of our tensor product of operads, one has $P \otimes C o m=P$ and hence the tensor product $A \otimes K$ of any $P$-algebra $A$ with a Com-algebra $K$ is always a $P$-algebra. This gives a bifunctor $\mathbf{A} \lg _{P} \times \mathbf{A} \lg _{\text {Com }} \rightarrow \mathbf{A} \lg _{P}$ defined on objects by $(A, K) \mapsto A \otimes K$.

2.5. Free algebras (c.f. $[\mathbf{L V}$, Section 5.2.5]. Let $P \in \mathbf{O p}$ be an operad and let $V$ be a graded vector space. If we forget the differential of $P$, the free $P$-algebra generated by $V$ is the $P$-algebra

$$
P\langle V\rangle=\bigoplus_{m \geq 0}\left(P(m) \otimes_{\Sigma_{m}} V^{\otimes m}\right)
$$

with the structure maps $\theta(m): P(m) \otimes_{\Sigma_{m}} P\langle V\rangle^{\otimes m} \rightarrow P\langle V\rangle$ given by the composition of the shuffle isomorphism followed by the structure morphisms $\gamma$ of $P$ :

$$
\begin{gathered}
P(l) \otimes\left(P\left(m_{1}\right) \otimes_{\Sigma_{m_{1}}} V^{\otimes m_{1}}\right) \otimes \cdots \otimes\left(P\left(m_{l}\right) \otimes_{\Sigma_{m_{l}}} V^{\otimes m_{l}}\right) \\
\cong v^{\downarrow S h} \\
P(l) \otimes\left(P\left(m_{1}\right) \otimes \cdots \otimes P\left(m_{l}\right)\right) \otimes \Sigma_{m_{1} \times \cdots \times \Sigma_{m_{l}}} V^{\otimes\left(m_{1}+\cdots+m_{l}\right)} \\
\downarrow^{\mid \gamma_{l ; m_{1}, \ldots, m_{l} \otimes 1}} \\
P\left(m_{1}+\cdots+m_{l}\right) \otimes V^{\otimes\left(m_{1}+\cdots+m_{l}\right)}
\end{gathered}
$$

By the universal property of the free $P$-algebra $[\mathbf{L V}]$, for any linear map $f: V \rightarrow A$ of degree 0 , there exists a unique morphism of $P$-algebras $P\langle V\rangle \rightarrow A$ that restricted to $V$ agrees with $f$. 
Remark 2.1. Note that the formula for the free $P$-algebra generated by $V$ also makes sense if $P$ carries a non-trivial differential. Also, it has the universal property of a free object in the category of $P$-dg algebras for graded vector spaces $V$ with zero differentials and $\mathbf{k}$-linear graded maps $f: V \rightarrow Z A$.

2.6. Cone of a morphism. Given a morphism $f: A \rightarrow B$ of $P$-algebras, we denote by $C(f)$ the cone of $f$. This is the cochain complex given by $C(f)^{n}=A^{n+1} \oplus B^{n}$ with differential $d(a, b)=(-d a,-f a+d b)$. The morphism $f$ is a quasi-isomorphism of $P$-algebras if and only if $H^{*}(C(f))=0$.

\section{Basic homotopy theory of operad algebras}

Throughout this section we let $P \in \mathbf{O p}$ be a fixed operad in the category of cochain complexes of vector spaces over $\mathbf{k}$. We first introduce KS-extensions of $P$-algebras and prove that they satisfy the lifting property with respect to surjective quasi-isomorphisms. Then, we give some main properties of homotopies between morphisms of $P$-algebras.

Remark 3.1. In general, in order to define extensions one would require the not easy notion of tensor product of $P$-algebras (see $[\mathbf{H i n 2}],[\mathbf{S U}]$, [MS], [Lod], for instance). Fortunately, in our case it suffices to consider tensor products of free (non-differential) algebras.

Definition 3.2. Let $n>0$ be an integer. Let $A=P\langle V\rangle \in \mathbf{A l g}_{P}$ be free as graded algebra. A degree $n K S$-extension of $A$ is the free graded $P$-algebra

$$
A \sqcup_{d} P\left\langle V^{\prime}\right\rangle:=P\left\langle V \oplus V^{\prime}\right\rangle,
$$

where $V^{\prime}$ is a graded vector space of homogeneous degree $n$ and $d: V^{\prime} \rightarrow$ $Z^{n+1}(A)$ a k-linear map. The differential on $A \sqcup_{d} P\left\langle V^{\prime}\right\rangle$ is defined as the only $P$-derivation extending $d$. This derivation squares to zero because so do its restrictions to $P, A$, and $V^{\prime}$.

We have the following universal property for KS-extensions:

Lemma 3.3. Let $A \sqcup_{d} P\left\langle V^{\prime}\right\rangle$ be a KS-extension of a free $P$-algebra $A=$ $P\langle V\rangle$, and let $f: A \rightarrow B$ be a morphism of P-algebras. A morphism $f^{\prime}$ : $A \sqcup_{d} P\left\langle V^{\prime}\right\rangle \rightarrow B$ extending $f$ is uniquely determined by a linear map $\varphi: V^{\prime} \rightarrow B$ of degree 0 satisfying $d \varphi=f d$.

Proof: By the universal property of free algebras we get $f^{\prime}: P\left\langle V \oplus V^{\prime}\right\rangle \rightarrow$ $B$. To prove that it is compatible with the differentials of $A \sqcup_{d} P\left\langle V^{\prime}\right\rangle$ and $B$, it suffices to check this on the restriction to $V^{\prime}$. We have $f^{\prime} \circ$ $\left.d\right|_{V^{\prime}}=f \circ d=d \circ \varphi=\left.d \circ f^{\prime}\right|_{V^{\prime}}$.

KS-extensions satisfy the lifting lemma with respect to surjective quasi-isomorphisms: 
Lemma 3.4. Let $i: A \rightarrow A \sqcup_{d} P\langle V\rangle$ be a KS-extension of degree $n$ and

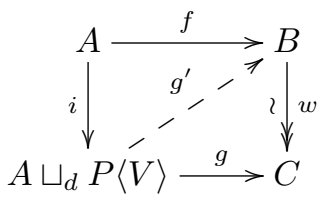

a solid commutative diagram of $P$-algebra morphisms, where $w$ is a surjective quasi-isomorphism. Then, there is a P-algebra morphism $g^{\prime}$ making both triangles commute.

Proof: Consider the solid diagram of $\mathbf{k}$-vector spaces

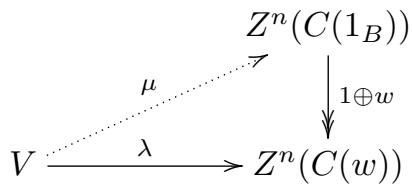

where $\lambda=\left(f \circ d,\left.g\right|_{V}\right)$. Since $w$ is a surjective quasi-isomorphism, this is well defined and $1 \oplus w$ is surjective. Therefore there exists a dotted arrow $\mu=(\alpha, \beta)$ making the diagram commute. It is straightforward to see that the image of the linear map $(d, \beta): V \rightarrow A^{n+1} \oplus B^{n}$ is included in $Z^{n}(C(f))$. According to the universal property of KS-extensions of Lemma 3.3, we may obtain $g^{\prime}$ as the morphism induced by $\left.g\right|_{A}$ together with $\beta: V \rightarrow B^{n}$.

Definition 3.5. A Sullivan P-algebra is the colimit $\mathcal{M}=\cup_{i \geq 0} \mathcal{M}[i]$ of a sequence

$\mathcal{M}[0]=P(0) \longrightarrow \mathcal{M}[1]=P\langle V[1]\rangle \longrightarrow \mathcal{M}[2]=\mathcal{M}[1] \sqcup_{d} P\langle V[2]\rangle \longrightarrow \cdots$

of KS-extensions of non-negative degrees, starting from $P(0)=P\langle 0\rangle$.

Proposition 3.6. Let $C$ be a Sullivan P-algebra. For every solid diagram of $P$-algebras

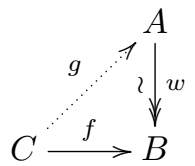

in which $w$ is a surjective quasi-isomorphism, there exists $g$ making the diagram commute.

Proof: Assume that $C^{\prime} \rightarrow C=C^{\prime} \sqcup_{d} P\langle V\rangle$ is a KS-extension of degree $n$, and that we have constructed $g^{\prime}: C^{\prime} \rightarrow A$ such that $w g^{\prime}=f^{\prime}$, where $f^{\prime}$ denotes the restriction of $f$ to $f^{\prime}$. The existence of $g$ extending $g^{\prime}$ now follows from Lemma 3.4. 
Remark 3.7. The above proposition says that Sullivan $P$-algebras are cofibrant objects in the Quillen model structure of the category of $P$-algebras of [Hin1]. In fact, Sullivan $P$-algebras correspond to the standard cofibrations of Hinich.

The following are standard consequences of Proposition 3.6. The proofs are straightforward adaptations of the analogous results in the setting of Com-algebras (see Section 11.3 of [GrMo], see also Section 2.3 of $[\mathbf{C i r}]$ for proofs in the abstract setting of categories with a functorial path).

Denote by $\mathbf{k}[t, d t]$ the Com-algebra with a generator $t$ in degree zero, a generator $d t$ in degree one, and $d(t)=d t$. We have the unit $\iota$ and evaluations $\delta^{0}$ and $\delta^{1}$ at $t=0$ and $t=1$ respectively, which are morphisms of Com-algebras satisfying $\delta^{0} \circ \iota=\delta^{1} \circ \iota=1$.

Definition 3.8. A functorial path in the category of $P$-algebras is defined as the functor

$$
-[t, d t]: \mathbf{A l g}_{P} \longrightarrow \mathbf{A l g}_{P}
$$

given on objects by $A[t, d t]=A \otimes \mathbf{k}[t, d t]$ and on morphisms by $f[t, d t]=$ $f \otimes \mathbf{k}[t, d t]$, together with the natural transformations

$$
A \stackrel{\iota}{\longrightarrow} A[t, d t] \underset{\delta^{0}}{\stackrel{\delta^{1}}{\longrightarrow}} A ; \quad \delta^{k} \circ \iota=1
$$

given by $\delta^{k}=1 \otimes \delta^{k}: A[t, d t] \rightarrow A \otimes \mathbf{k}=A$ and $\iota=1 \otimes \iota: A=A \otimes \mathbf{k} \rightarrow$ $A[t, d t]$.

Note that the map $\iota$ is a quasi-isomorphism of $P$-algebras while the maps $\delta^{0}$ and $\delta^{1}$ are surjective quasi-isomorphisms of $P$-algebras.

The functorial path gives a natural notion of homotopy between morphisms of $P$-algebras:

Definition 3.9. Let $f, g: A \rightarrow B$ be two morphisms of $P$-algebras. An homotopy from $f$ to $g$ is given by a morphism of $P$-algebras $h: A \rightarrow$ $B[t, d t]$ such that $\delta^{0} \circ h=f$ and $\delta^{1} \circ h=g$. We use the notation $h: f \simeq g$.

The homotopy relation defined by a functorial path is reflexive and compatible with the composition (see for example [KP, Lemma I.2.3]. Furthermore, the symmetry of Com-algebras $\mathbf{k}[t, d t] \rightarrow \mathbf{k}[t, d t]$ given by $t \mapsto 1-t$ makes the homotopy relation into a symmetric relation. However, the homotopy relation is not transitive in general. As in the rational homotopy setting of Com-algebras, we have:

Proposition 3.10. The homotopy relation between morphisms of $P$-algebras is an equivalence relation for those morphisms whose source is a Sullivan P-algebra. 
Proof: It only remains to prove transitivity. Let $C$ be a Sullivan $P$-algebra and consider morphisms $f, f^{\prime}, f^{\prime \prime}: C \rightarrow A$ together with homotopies $h: f \simeq f^{\prime}$ and $h^{\prime}: f^{\prime} \simeq f^{\prime \prime}$. Consider the pull-back diagram in the category of $P$-algebras

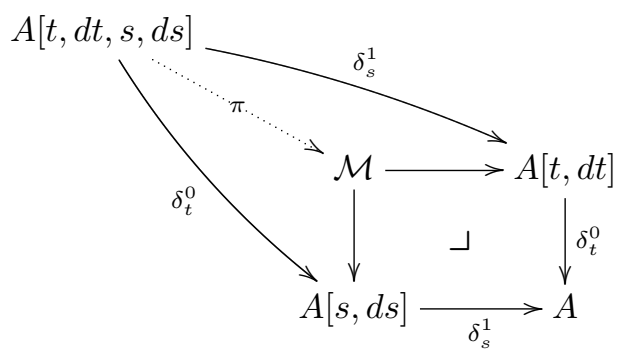

To see that the map $\pi$ is surjective, note that if $a(s, d s)$ and $b(t, d t)$ are polynomials such that $a(1,0)=b(0,0)$, representing an element in $\mathcal{M}$, then

$$
\pi(a(s, d s)+b(s t, d t)-b(0,0))=(a(s, d s), b(t, d t)) .
$$

It is straightforward to see that all the $P$-algebras in the above diagram are quasi-isomorphic and that $\pi$ is a quasi-isomorphism. Consider the solid diagram

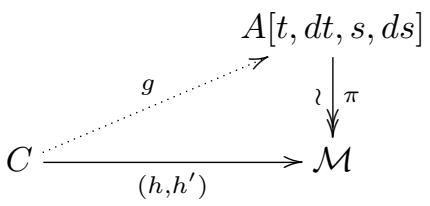

Then by Proposition 3.6, there exists a dotted arrow $g$ such that $\pi g=$ $\left(h, h^{\prime}\right)$. Let $h \widetilde{+} h^{\prime}:=\nabla g$, where $\nabla: A[t, d t, s, d s] \rightarrow A[t, d t]$ is the map given by $t, s \mapsto t$. This gives the desired homotopy $h \widetilde{+} h^{\prime}: f \simeq f^{\prime \prime}$.

Denote by $[A, B]$ the set of homotopy classes of morphisms of $P$-algebras $f: A \rightarrow B$.

Proposition 3.11. Let $C$ be a Sullivan P-algebra. Any quasi-isomorphism $w: A \rightarrow B$ of $P$-algebras induces a bijection $w_{*}:[C, A] \rightarrow[C, B]$.

Proof: We first prove surjectivity. Consider the mapping path of $w$, given by the pull-back

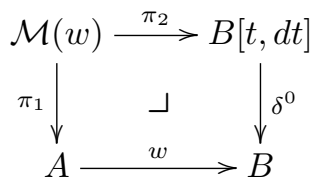


Define maps $p:=\pi_{1}: \mathcal{M}(w) \rightarrow A, q:=\delta^{1} \pi_{2}: \mathcal{M}(w) \rightarrow B$, and $j:=$ $(1, \iota w): A \rightarrow \mathcal{M}(w)$. We obtain a solid diagram

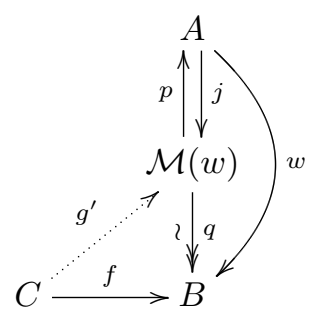

where $q$ is a surjective quasi-isomorphism and $q j=w$. By Proposition 3.6, there exists $g^{\prime}$ such that $q g^{\prime}=f$. Let $g:=p g^{\prime}$. Then we have $f=q g^{\prime}=\delta^{1} \pi_{2} g^{\prime}$ and $w g=w \pi_{1} g^{\prime}=\delta^{0} \pi_{2} g^{\prime}$. Therefore $[w g]=[f]$ and $w_{*}$ is surjective.

To prove injectivity, let $f_{0}, f_{1}: C \rightarrow A$ be such that $h: w f_{0} \simeq w f_{1}$. Consider the pull-back diagram

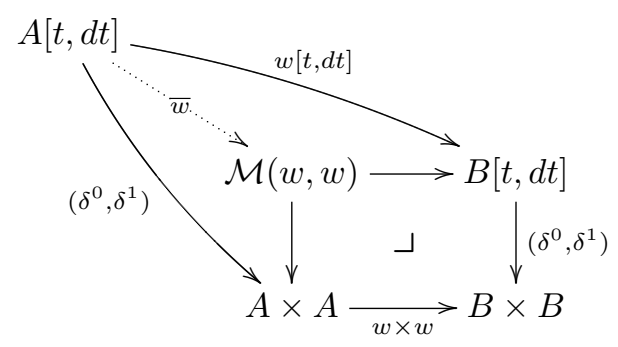

One may verify that $\bar{w}$ is a quasi-isomorphism. Let $H=\left(f_{0}, f_{1}, h\right)$ and consider the solid diagram

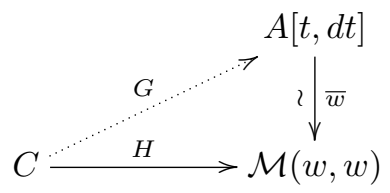

Since $\bar{w}_{*}$ is surjective, there exists a dotted arrow $G$ such that $\bar{w} G \simeq H$. It follows that $f_{0} \simeq \delta^{0} G \simeq \delta^{1} G \simeq f_{1}$. Then $f_{0} \simeq f_{1}$ by Proposition 3.10.

\section{Sullivan minimal models}

In this section, we prove the existence of Sullivan minimal models of $P$-algebras, for a quite wide family of operads in the category of cochain complexes of $\mathbf{k}$-vector spaces.

We first introduce the notion of $r$-tame operad. For this class of operads, $r$-connected $P$-algebras will have Sullivan minimal models. 
Definition 4.1. Let $r \geq 0$ be an integer. An operad $P \in \mathbf{O p}$ is called $r$-tame if for all $n \geq 2$,

$$
P(n)^{q}=0 \text { for all } q \leq(1-n)(1+r) .
$$

Note that $r$-tame implies $(r+1)$-tame for all $r \geq 0$. Below we represent the condition for being an $r$-tame operad, for $r=0$ and $r=1$. Elements of $r$-tame operads are allowed to be non-zero in the arity-degree range determined by the blank squares below, except for the identity id $\in$ $P(1)=\mathbf{k}$, and $P(0) \in\{0, \mathbf{k}\}$ which are denoted by $*$ and live in aritydegree $(1,0)$ and $(0,0)$ respectively.
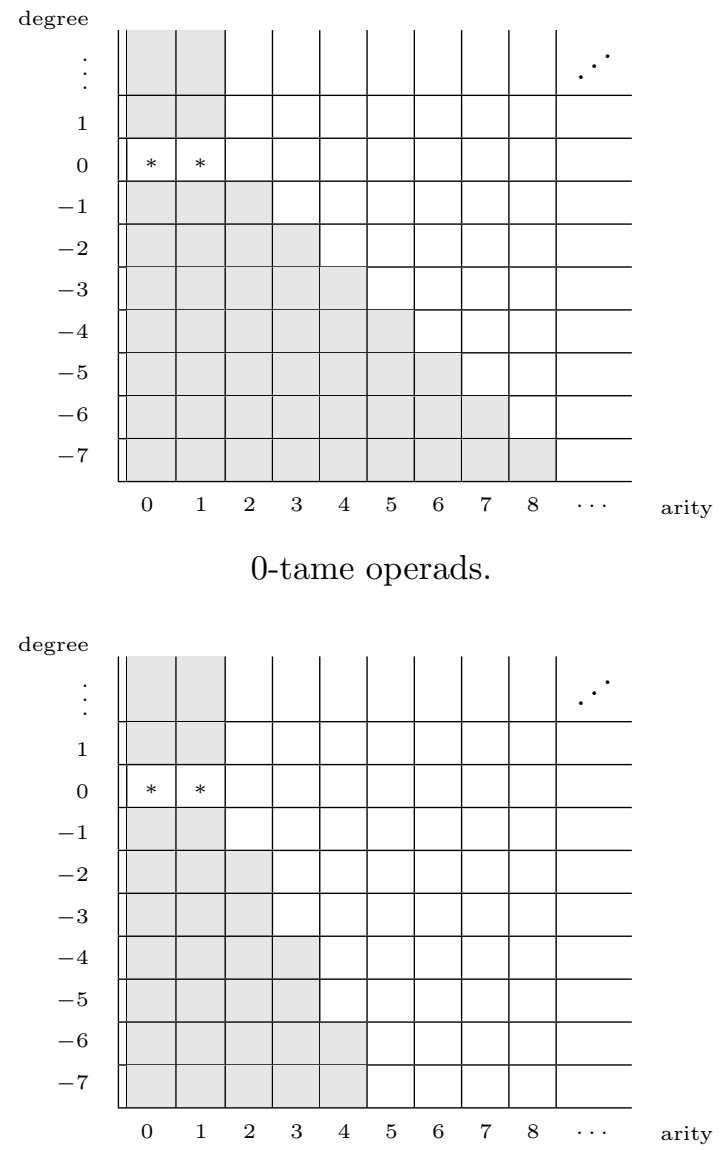

1-tame operads. 
Definition 4.2. Let $r \geq 0$ be an integer. A Sullivan $r$-minimal $P$-algebra is the colimit $\mathcal{M}=\cup_{i>0} \mathcal{M}[i]$ of a sequence of KS-extensions starting from $P(0)$, ordered by non-decreasing degrees bigger than $r$ :

$\mathcal{M}[0]=P(0) \longrightarrow \mathcal{M}[1]=P\langle V[1]\rangle \longrightarrow \mathcal{M}[2]=\mathcal{M}[1] \sqcup_{d} P\langle V[2]\rangle \longrightarrow \cdots$

with $r<\operatorname{deg}(V[n]) \leq \operatorname{deg}(V[n+1])$ for all $n \geq 1$. A Sullivan $r$-minimal model for a $P$-algebra $A$ is a Sullivan $r$-minimal $P$-algebra $\mathcal{M}$ together with a quasi-isomorphism $f: \mathcal{M} \rightarrow A$.

As in the rational homotopy setting, to prove the existence of Sullivan minimal models we will restrict to the case when our $P$-algebras are cohomologically connected (which we will call connected for short from now on).

Definition 4.3. A $P$-algebra $A$ is called 0 -connected if $H^{i}(A)=0$ for all $i<0$ and the unit map $\eta: P(0) \rightarrow A$ induces an isomorphism $P(0) \cong$ $H^{0}(A)$. Let $r \geq 0$. Then $A$ is called $r$-connected if, in addition, $H^{1}(A)=$ $\cdots=H^{r}(A)=0$.

For the construction of Sullivan minimal models we will use the following two lemmas. The first of these lemmas ensures that free $P$-algebras generated by positively-graded vector spaces, are positively-graded when $P$ is tame.

Lemma 4.4. Let $V=\bigoplus_{i>r} V^{i}$ be a graded vector space with degrees $>r$. If $P$ is $r$-tame then $P\langle V\rangle^{0}=P(0)$ and $P\langle V\rangle^{k}=0$ for all $k \leq r$ with $k \neq 0$. In particular, $P\langle V\rangle$ is r-connected.

Proof: Let $k \in \mathbb{Z}$. The degree $k$-part of $P\langle V\rangle$ may be written as

$$
\begin{aligned}
& P\langle V\rangle^{k}=P(0)^{k} \oplus\left(\sum_{i>r} P(1)^{k-i} \otimes_{\Sigma_{1}} V^{i}\right) \\
& \oplus\left(\sum_{\substack{n \geq 2, i_{1}, \ldots, i_{n}>r}} P(n)^{q_{n}} \otimes_{\Sigma_{n}} V^{i_{1}} \otimes \cdots \otimes V^{i_{n}}\right),
\end{aligned}
$$

where $q_{n}=k-i_{1}-\cdots-i_{n} \leq k-n(1+r)$. Since $P(0)^{k}=0$ for all $k \neq 0$ and $P(1)^{k-i}=0$ for all $k \neq i$, it suffices to see that for all $n \geq 2$ and all $k \leq r$ we have $P(n)^{q_{n}}=0$. Since $P$ is $r$-tame, it suffices to prove that $q_{n} \leq q_{n}^{*}:=(1-n)(1+r)$. Let $n \geq 2$ be fixed and assume that $k \leq r$. Then $q_{n}=k-i_{1}-\cdots-i_{n} \leq k-n(1+r) \leq r-n(1+r)=q_{n}^{*}-1<q_{n}^{*}$.

The second lemma characterizes the good behavior of $r$-tame operads with respect to KS-extensions and is inspired in Lemma 10.4 of [GrMo]. 
Lemma 4.5. Let $V=\bigoplus_{r<i<p} V^{i}$ be a graded vector space with $0 \leq r<$ $i \leq p$. Let $V^{\prime}$ be a graded vector space of homogeneous degree $p$ and let $P$ be an $r$-tame operad. Then:

(1) $P\left\langle V \oplus V^{\prime}\right\rangle^{k}=P\langle V\rangle^{k}$ for all $k<p$ and $P\left\langle V \oplus V^{\prime}\right\rangle^{p}=P\langle V\rangle^{p} \oplus V^{\prime}$.

(2) If $r+1<p$ and $V^{r+1}=0$ then $P\left\langle V \oplus V^{\prime}\right\rangle^{p+1}=P\langle V\rangle^{p+1}$.

Proof: For all $k \in \mathbb{Z}$ we may write

$$
\begin{aligned}
\frac{P\left\langle V \oplus V^{\prime}\right\rangle^{k}}{P\langle V\rangle^{k}}= & \left(\sum_{n \geq 1} P(n)^{q_{n}} \otimes_{\Sigma_{n}} V^{\prime \otimes n}\right) \\
& \oplus\left(\sum_{\substack{n \geq 2,1 \leq j \leq n-1 \\
r<i_{1} \leq \cdots \leq i_{j} \leq p}} P(n)^{q_{n}^{\prime}} \otimes_{\Sigma_{n}} V^{i_{1}} \otimes \cdots \otimes V^{i_{j}} \otimes V^{\prime \otimes(n-j)}\right),
\end{aligned}
$$

where $q_{n}=k-p n$ and $q_{n}^{\prime}=k-i_{1}-\cdots-i_{j}-p(n-j)$. We first show that for $n \geq 2$ and $k \leq p$, we have $P(n)^{q_{n}}=P(n)^{q_{n}^{\prime}}=0$. Since $P$ is $r$-tame, it suffices to see that both $q_{n}$ and $q_{n}^{\prime}$ are smaller or equal than $q_{n}^{*}:=(1-n)(r+1)$. Since $r<p$, we have

$$
q_{n}=k-p n \leq p(1-n) \leq(1-n)(1+r)=q_{n}^{*} .
$$

Note that $q_{n}^{\prime}$ attains its maximum when $k=p, j=n-1$, and $i_{1}=\cdots=$ $i_{j}=r+1$. Then

$$
q_{n}^{\prime} \leq p+(1-n)(1+r)-p=q_{n}^{*} .
$$

This proves that for $k \leq p$ we have

$$
\frac{P\left\langle V \oplus V^{\prime}\right\rangle^{k}}{P\langle V\rangle^{k}} \cong P(1)^{k-p} \otimes V^{\prime} .
$$

Now (1) follows from the fact that $P(1)^{k-p}=0$ for all $k \neq p$ and $P(1)^{0}=\mathbf{k}$.

Assume that $p>r+1$ and $V^{r+1}=0$. Then in the above formula for $P\left\langle V \oplus V^{\prime}\right\rangle^{p+1} / P\langle V\rangle^{p+1}$ we have: if $n>1$ then

$$
q_{n}=p+1-p n=p(1-n)+1 \leq(r+2)(1-n)+1=q_{n}^{*}+2-n \leq q_{n}^{*}
$$
and $q_{1}=1 \neq 0$.

Note that now $q_{n}^{\prime}$ attains its maximum when $j=n-1$ and $i_{1}=\cdots=$ $i_{j}=r+2$. Then for all $n \geq 2$ we have

$q_{n}^{\prime}=p+1-i_{1}-\cdots-i_{j}-p(n-j) \leq p+1+(r+2)(1-n)-p=q_{n}^{*}+(2-n) \leq q_{n}^{*}$.

Therefore all the contributions vanish and (2) is satisfied. 
Theorem 4.6. Let $P$ be an $r$-tame operad. Then every $r$-connected $P$-algebra $A$ has a Sullivan $r$-minimal model $f: \mathcal{M} \rightarrow A$ with $\mathcal{M}^{0}=$ $P(0)$ and $\mathcal{M}^{i}=0$ for all $i<r$ with $i \neq 0$. Furthermore, if $A$ is $(r+1)$-connected and $H^{*}(A)$ is of finite type, then $\mathcal{M}$ is of finite type.

Proof: We follow the steps of the classical proof of existence of Sullivan minimal models for Com-algebras (see [GrMo, Theorem 10.3] for the case of simply connected Com-algebras and [GrMo, Theorem 13.1] or [GeMa, Theorem V.8.11] for the non-simply connected case).

We will construct, inductively over the degree $n \geq 0$, a sequence of free $P$-algebras $\mathcal{M}[n]$ together with morphisms of $P$-algebras $f_{n}: \mathcal{M}[n] \rightarrow A$ satisfying the following conditions:

$\left(a_{n}\right)$ The $P$-algebra $\mathcal{M}[n]$ Sullivan $r$-minimal and is either equal to $\mathcal{M}[n-1]$ or a composition of KS-extensions of degree $n$ of $\mathcal{M}[n-1]$. The morphism $f_{n}$ extends $f_{n-1}$.

$\left(b_{n}\right)$ The map $H^{i} f_{n}$ is an isomorphism for all $i \leq n$ and a monomorphism for $i=n+1$.

Then the morphism $f: \cup_{n} f_{n}: \cup_{n} \mathcal{M}[n] \rightarrow A$ will be a Sullivan $r$ minimal model for $A$. Indeed, condition $\left(a_{n}\right)$ implies that $\mathcal{M}$ is Sullivan $r$-minimal and that $\mathcal{M}^{n}=\mathcal{M}[k]^{n}$ for all $k \geq n$. From $\left(b_{n+1}\right)$ it follows that $H^{n}(C(f))=H^{n}\left(C\left(f_{n+1}\right)\right)=0$. Therefore $f$ is a quasi-isomorphism.

Let $\mathcal{M}[0]=P(0)$. The unit map $\eta: P(0) \rightarrow A$ gives a morphism of $P$-algebras $f_{0}: \mathcal{M}[0] \rightarrow A$. For all $0<i \leq r$ we let $\mathcal{M}[i]=\mathcal{M}[0]$ and $f_{i}=f_{0}$. Since $A$ is $r$-connected, conditions $\left(a_{i}\right)$ and $\left(b_{i}\right)$ are satisfied for all $i \leq r$.

Assume inductively that we have a morphism of $P$-algebras $f_{n-1}$ : $\mathcal{M}[n-1] \rightarrow A$ satisfying $\left(a_{n-1}\right)$ and $\left(b_{n-1}\right)$. Condition $\left(b_{n-1}\right)$ is equivalent to the vanishing of $H^{i}\left(C\left(f_{n-1}\right)\right)$ for all $i<n$. Let

$$
V[n, 0]:=H^{n}\left(C\left(f_{n-1}\right)\right)
$$

and consider it as a graded vector space of homogeneous degree $n$. Take a section of the projection $Z^{n}\left(C\left(f_{n-1}\right)\right) \rightarrow V[n, 0]$ to obtain a linear differential $d: V[n, 0] \rightarrow Z^{n+1} \mathcal{M}[n-1]$ and a linear map $\varphi: V[n, 0] \rightarrow A^{n}$ such that $d \varphi=f_{n-1} d$. We then let

$$
\mathcal{M}[n, 0]:=\mathcal{M}[n-1] \sqcup_{d} P\langle V[n, 0]\rangle
$$

and denote by $f_{n, 0}: \mathcal{M}[n, 0] \rightarrow A$ the extension of $f_{n-1}$ by $\varphi$.

By Lemma $4.4, \mathcal{M}[n, 0]$ is an $r$-connected $P$-algebra. Furthermore, by (1) of Lemma 4.5 we have that $\mathcal{M}[n, 0]^{k}=\mathcal{M}[n-1]^{k}$ for all $k<n$ and $\mathcal{M}[n, 0]^{n}=\mathcal{M}[n-1]^{n} \oplus V[n, 0]$. In particular, we have $H^{i} f_{n, 0}=$ $H^{i} f_{n-1}$ for all $i<n$. Hence by induction hypothesis, $H^{i} f_{n, 0}$ is an isomorphism for all $i<n$. We next prove that $H^{n} f_{n, 0}$ is an isomorphism. 
Denote by $j_{0}: \mathcal{M}[n-1] \rightarrow \mathcal{M}[n, 0]$ the inclusion. The morphism of cones (id, $\left.f_{n, 0}\right): C\left(j_{0}\right) \rightarrow C\left(f_{n-1}\right)$ induces an isomorphism in degree $n$ cohomology

$$
H^{n}\left(\mathrm{id}, f_{n, 0}\right): H^{n}\left(C\left(j_{0}\right)\right) \rightarrow H^{n}\left(C\left(f_{n-1}\right)\right) .
$$

Indeed, since $\mathcal{M}[n, 0]^{n}=\mathcal{M}[n-1]^{n} \oplus V[n, 0]$, every element in $Z^{n}\left(C\left(j_{0}\right)\right)$ may be written as $\left(x, x^{\prime}+v\right)$ where $x, x^{\prime} \in \mathcal{M}[n-1]$ and $v \in V[n, 0]$ are such that $d x=0$ and $d x^{\prime}+d v=x$. The map $H^{n}\left(\mathrm{id}, f_{n, 0}\right)$ is then given by

$$
\left[\left(x, x^{\prime}+v\right)\right] \longmapsto\left[\left(x, f_{n-1} x^{\prime}+\varphi v\right)\right] .
$$

To prove surjectivity, note that if $[(x, a)] \in H^{n}\left(C\left(f_{n-1}\right)\right)$, then there exists $v \in V[n, 0]$ with $d v=x$ and $\varphi v=a$. Therefore $[(x, v)] \in$ $H^{n}\left(C\left(j_{0}\right)\right)$ maps to $[(x, a)]$. To prove injectivity, note that every element in $H^{n}\left(C\left(j_{0}\right)\right)$ admits a representative of the form $(d v, v)$. Then the condition $(d v, \varphi v)=D(x, a)=\left(d x, f_{n-1} x-d a\right)$ implies that $v=0$.

Now, consider the morphism of long exact sequences in cohomology

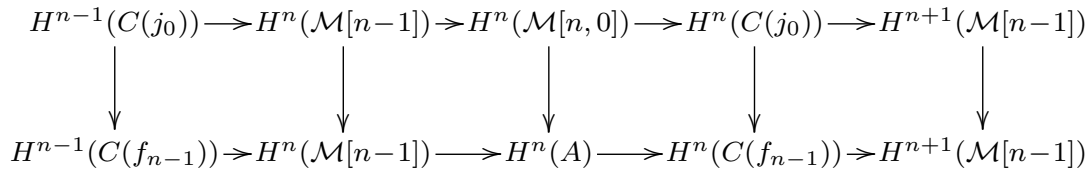

Since $H^{n-1}\left(C\left(j_{0}\right)\right)=0$ and $H^{n}$ (id, $\left.f_{n, 0}\right)$ is an isomorphism, it follows from the five lemma that $H^{n} f_{n, 0}$ is an isomorphism.

To make $H^{n+1} f_{n, 0}$ into a monomorphism, let

$$
V[n, 1]:=\operatorname{Ker}\left(H^{n+1} f_{n, 0}\right)=H^{n}\left(C\left(f_{n, 0}\right)\right)
$$

and

$$
\mathcal{M}[n, 1]=\mathcal{M}[n, 0] \sqcup_{d} P\langle V[n, 1]\rangle,
$$

where $V[n, 1]$ is considered as a vector space of homogeneous degree $n$ and as in the previous step, we take a section of the projection $Z^{n}\left(C\left(f_{n, 0}\right)\right) \rightarrow$ $V[n, 1]$ to define a differential on $V[n, 1]$ and a map $f_{n, 1}: \mathcal{M}[n, 1] \rightarrow A$.

Denote by $j_{1}: \mathcal{M}[n, 0] \rightarrow \mathcal{M}[n, 1]$ the inclusion. Let $[x] \in H^{n+1}(\mathcal{M}[n, 0])$. If $[x] \in \operatorname{Ker}\left(H^{n+1} f_{n, 0}\right)$ then we may write $f_{n, 0} x=d a$ for some $a \in A$. The pair $[(x, a)]$ gives an element $v \in \mathcal{M}[n, 1]$ with $d v=x$. This proves that we have an inclusion

$$
\operatorname{Ker}\left(H^{n+1} f_{n, 0}\right) \subset \operatorname{Ker}\left(H^{n+1} j_{1}\right) .
$$

We iterate the above process by letting

$$
V[n, i]=\operatorname{Ker}\left(H^{n+1} f_{n, i-1}\right)=H^{n}\left(C\left(f_{n, i-1}\right)\right)
$$

and

$$
\mathcal{M}[n, i]=\mathcal{M}[n, i-1] \sqcup_{d} P\langle V[n, i]\rangle,
$$


until $\operatorname{Ker}\left(H^{n+1} f_{n, i}\right)=0$. If this never happens, we let $\mathcal{M}[n]:=\cup_{i} \mathcal{M}[n, i]$ and define $f_{n}: \mathcal{M}[n] \rightarrow A$ by $\left.f_{n}\right|_{\mathcal{M}[n, i]}=f_{n, i}$. Reasoning as before, we obtain an inclusion

$$
\operatorname{Ker}\left(H^{n+1} f_{n, i}\right) \subset \operatorname{Ker}\left(H^{n+1} j_{i+1}\right),
$$

where $j_{i}: \mathcal{M}[n, i-1] \rightarrow \mathcal{M}[n, i]$ denotes the inclusion. Let $x \in \operatorname{Ker}\left(H^{n+1} f_{n}\right)$. Then it has a representative $x_{i} \in H^{n+1} f_{n, i}$ for some $i$. But then the inclusion $\operatorname{Ker}\left(H^{n+1} f_{n, i}\right) \subset \operatorname{Ker}\left(H^{n+1} j_{i+1}\right)$ implies that the image of $x_{i}$ in $H^{n+1} f_{n, i+1}$ is trivial. Hence $x=0$. This proves that $\operatorname{Ker}\left(H^{n+1} f_{n}\right)=$ 0 . Since $H^{i} f_{n, i}$ is an isomorphism for each $i \leq n$, it follows that $H^{n} f_{n}$ is an isomorphism. Therefore $\left(b_{n}\right)$ is satisfied. This ends the inductive step.

If $A$ is $(r+1)$-connected, then we can take $\mathcal{M}[r+1]=\mathcal{M}[r]$ and $\left(a_{r+1}\right)$ and $\left(b_{r+1}\right)$ are satisfied. For $n>r+1$, by Lemma 4.5 we have that $\mathcal{M}[n, 0]^{n+1}=\mathcal{M}[n-1]^{n+1}$. This implies that $\operatorname{Ker}\left(H^{n+1} f_{n, 0}\right)=0$ and hence $\mathcal{M}[n]=\mathcal{M}[n, 0]=\mathcal{M}[n-1] \sqcup_{d} P\langle V[n, 0]\rangle$. If $H^{*}(A)$ has finite type, then $V[n, 0]$ is finite dimensional and $\mathcal{M}[n]$ has finite type.

Let us review a few examples where Theorem 4.6 applies.

The operads $\mathcal{A} s s$, Com, and Lie encoding differential graded associative, commutative and Lie algebras respectively are generated by operations in arity-degree $(2,0)$. Therefore they are concentrated in degree 0 . We have:

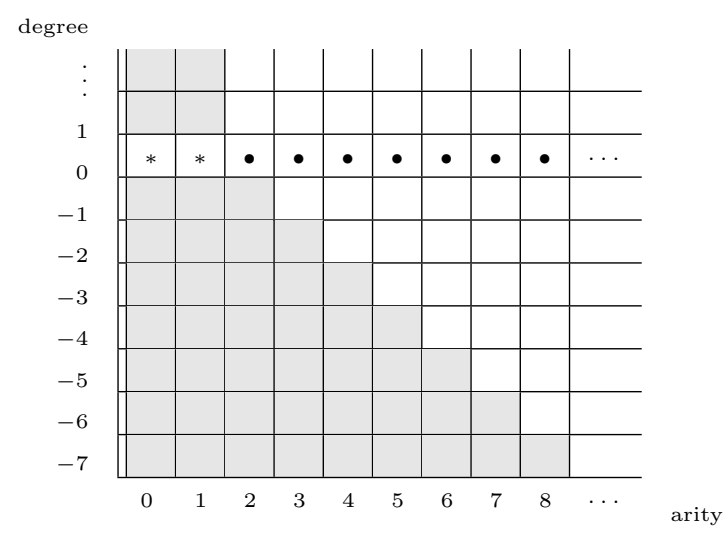

The operads $\mathcal{A} s s$, Com, and Lie are 0-tame. 
The above operads have minimal models, encoding the infinity-versions of their algebras. These are depicted in the following table.

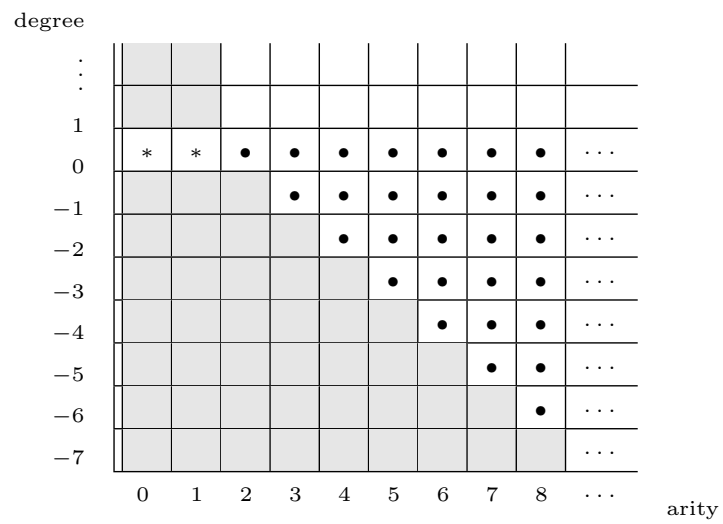

The operads $\mathcal{A} s s_{\infty}, \operatorname{Com}_{\infty}$, and $\mathcal{L} i e_{\infty}$ are 0 -tame.

Corollary 4.7. Let $P$ be one of the operads Ass, Com, Lie, Com ${ }_{\infty}$, $\mathcal{A} s s_{\infty}$, or $\mathcal{L} i e_{\infty}$. Then every 0 -connected P-algebra has a Sullivan minimal model. Also, every 1-connected P-algebra with finite type cohomology has a Sullivan minimal model of finite type.

More generally, every reduced operad $P$ such that $H(P)(1)=\mathbf{k}$ has a minimal model (see Theorem 3.125 in [MSS]). We next prove that minimal models of reduced $r$-tame operads are $r$-tame. We first introduce some notation.

Definition 4.8. Let $P \in \mathbf{O p}$. Given $w \in P(n)^{q}$, we will denote by $|w|:=(n, q)$ its arity-degree. We will say that $w$ is $r$-tame if $q>(r+$ $1)(1-n)$. Note that $P$ is $r$-tame if and only if all its non-trivial elements of arity $\geq 2$ are $r$-tame.

Lemma 4.9. Every free operad $P \in \mathbf{O p}$ generated by $r$-tame elements is $r$-tame.

Proof: It suffices to show that if $w, w^{\prime} \in P$ are $r$-tame, then their partial compositions $w \circ_{i} w^{\prime}$ are also $r$-tame. Let $|w|=(n, q)$ and $\left|w^{\prime}\right|=\left(n^{\prime}, q^{\prime}\right)$. Then

$q+q^{\prime} \geq(r+1)(1-n)+1+(r+1)\left(1-n^{\prime}\right)+1=(r+1)\left(1-\left(n+n^{\prime}-1\right)\right)+2$. Since $\left|w \circ_{i} w^{\prime}\right|=\left(n+n^{\prime}-1, q+q^{\prime}\right)$, this implies that $w \circ_{i} w^{\prime}$ is $r$-tame. 
Proposition 4.10. Let $P \in \mathbf{O p}$ be a reduced $r$-tame operad. Then its minimal model is $r$-tame.

Proof: From the construction of minimal models of Theorem 3.125 in [MSS], we easily deduce that for any reduced operad $P$ with $H(P)(1)=$ $\mathbf{k}$, there is a minimal model $M \rightarrow P$ where $M=\cup_{n \geq 2} M_{n}$ is constructed inductively over the arity $n$ and satisfies:

(i) $M_{2}$ is freely generated by elements of $P(2)$, with $M_{2}(0)=0$ and $M_{2}(1)=\mathbf{k}$.

(ii) For $n>2, M_{n}$ is obtained as a free extension of $M_{n-1}$ by subspaces $A(n, q) \subseteq P(n)^{q}$ in arity-degree $(n, q)$ and subspaces $B(n, q) \subseteq$ $M_{n-1}(n)^{q}$ in arity-degree $(n, q-1)$.

For our purposes, it is not necessary to know neither which elements we are adding nor what are their differentials. We only need to keep track of their possible arities and degrees.

If $P$ is $r$-tame, then $M_{2}$ is clearly $r$-tame. Assume inductively that $M_{i}$ is $r$-tame for all $i<n$. Property (ii) tells us that $M_{n}$ is obtained as a free extension of $M_{n-1}$ by subspaces $A(n, q) \subseteq P(n)^{q}$ in aritydegree $(n, q)$ and subspaces $B(n, q) \subseteq M_{n-1}(n)^{q}$ in arity-degree $(n, q-1)$. Elements in $A(n, q)$ are clearly $r$-tame. Let $w \in B(n, q)$. Since $M_{n-1}$ is generated by elements in arity $<n$, we may write $w$ as a sum of partial compositions of the form $w^{\prime} \circ_{i} w^{\prime \prime}$ where $w^{\prime}$ and $w^{\prime \prime}$ are $r$-tame elements of $M_{n-1}$. Let $\left|w^{\prime}\right|=\left(n^{\prime}, q^{\prime}\right)$ and $\left|w^{\prime \prime}\right|=\left(n^{\prime \prime}, q^{\prime \prime}\right)$. Then $\left|w^{\prime} \circ_{i} w^{\prime \prime}\right|=$ $\left(n^{\prime}+n^{\prime \prime}-1, q^{\prime}+q^{\prime \prime}\right)=(n, q)$ We get:

$$
\begin{aligned}
q=q^{\prime}+q^{\prime \prime} \geq & (r+1)\left(1-n^{\prime}\right)+1+(r+1)\left(1-n^{\prime \prime}\right)+1 \\
& =(r+1)\left(1-\left(n^{\prime}+n^{\prime \prime}-1\right)\right)+2=(r+1)(1-n)+2 .
\end{aligned}
$$

This gives $q-1>(r+1)(1-n)$, which is precisely the condition for $w$ to be $r$-tame. This proves that $M_{n}$ is $r$-tame and hence $M$ is also $r$-tame.

Corollary 4.11. Let $P \in \mathbf{O p}$ be a reduced $r$-tame operad and let $P_{\infty} \rightarrow$ $P$ be a minimal model of $P$. Then every $r$-connected $P_{\infty}$-algebra has a Sullivan $r$-minimal model. Also, every $(r+1)$-connected $P_{\infty}$-algebra with finite type cohomology has a Sullivan $r$-minimal model of finite type.

An example of 1-tame operad is given by the operad encoding Gerstenhaber algebras: these are graded-commutative algebras with a Lie bracket of degree -1 satisfying the Poisson identity. The ordinary multiplication has arity-degree $(2,0)$, while the Lie bracket has arity-degree $(2,-1)$. We have: 


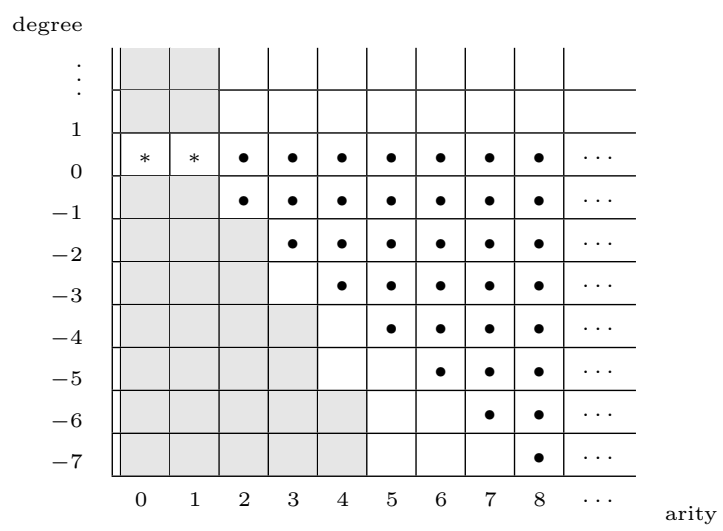

The Gerstenhaber operad Ger is 1-tame.

Corollary 4.12. Every 1-connected Ger-algebra (resp. Ger $\infty_{- \text {-algebra) }}$ has a Sullivan minimal model and every 2-connected Ger-algebra (resp. $\mathrm{C}_{\mathrm{C}} \mathrm{s}_{\infty}$-algebra) with finite type cohomology has a Sullivan minimal model of finite type.

Remark 4.13. In the last section of this paper, we will study chain $P$-algebras for operads of chain complexes; that is, both with positive homological degrees and differential of degree -1 . In this setting, the generator corresponding to the Lie bracket in the Gerstenhaber operad has arity-degree $(2,1)$. In particular, Ger is a 0 -tame operad and the restriction to 1-connected algebras is no longer necessary.

\section{Uniqueness of the minimal model}

In this section we prove the uniqueness of Sullivan minimal models. The proof is parallel to that in the setting of Com-algebras. As in the previous section, the key ingredient is Lemma 4.5.

Lemma 5.1. Let $P$ be an $r$-tame operad and let $f: A \rightarrow \mathcal{M}$ be a quasiisomorphism of $r$-connected $P$-algebras, with $\mathcal{M}$ a Sullivan $r$-minimal $P$-algebra. Then there exists a morphism of P-algebras $g: \mathcal{M} \rightarrow A$ such that $f g=\mathrm{id}_{\mathcal{M}}$.

Proof: We rewrite the proof of Gómez Tato (see Lemma 4.4 of [Góm]) for Com-algebras in the $P$-algebra setting (see also Theorem 14.11 of [FHT] and [Roi3], [Roi1] for a categorical version).

By definition, we may write $\mathcal{M}=\mathcal{M}^{\prime} \sqcup_{d} P\langle V\rangle$ where $\mathcal{M}^{\prime}$ is a free $P$-algebra generated by a graded vector space $V^{\prime}$ of degrees $r<i \leq p$ 
and $V$ is a graded vector space of homogeneous degree $p$, with $p>0$. Assume inductively that we have a morphism of $P$-algebras $g^{\prime}: \mathcal{M}^{\prime} \rightarrow A$ such that $f g^{\prime}=1_{\mathcal{M}^{\prime}}$. Then $g^{\prime}$ is injective. The morphism $f$ induces a morphism of cochain complexes (not of $P$-algebras!)

$$
\bar{f}: A / g^{\prime}\left(\mathcal{M}^{\prime}\right) \longrightarrow \mathcal{M} / \mathcal{M}^{\prime}
$$

which is a quasi-isomorphism. By Lemma 4.5 we have that $\left(\mathcal{M} / \mathcal{M}^{\prime}\right)^{p-1}=$ 0 and that $\left(\mathcal{M} / \mathcal{M}^{\prime}\right)^{p}=V$. This gives a surjection at the level of cocycles

$$
\pi: Z^{p}\left(A / g^{\prime}\left(\mathcal{M}^{\prime}\right)\right) \longrightarrow Z^{p}\left(\mathcal{M} / \mathcal{M}^{\prime}\right)=V .
$$

We obtain a linear map $\varphi: V \rightarrow A^{p}$ such that $f \varphi=1_{V}$ to a morphism $g: \mathcal{M} \rightarrow A$ by taking sections of the projections $A \rightarrow A / g^{\prime}\left(\mathcal{M}^{\prime}\right)$ and $\pi$ and considering the composition

$$
V=\left(\mathcal{M} / \mathcal{M}^{\prime}\right)^{p} \longrightarrow Z^{p}\left(A / g^{\prime}\left(\mathcal{M}^{\prime}\right) \longleftrightarrow\left(A / g^{\prime}\left(\mathcal{M}^{\prime}\right)\right)^{p} \longrightarrow A^{p}\right.
$$

For a proof of this last fact taking elements and checking that everything works fine see the proof of Theorem 14.11 in [FHT]. By Lemma 3.3, the map $\varphi$ extends $f^{\prime}$ to a morphism $f: \mathcal{M} \rightarrow A$.

As a classical consequence of Lemma 5.1 we have:

Lemma 5.2. Let $P$ be an $r$-tame operad and let $f: \mathcal{M} \rightarrow \mathcal{M}^{\prime}$ be a quasi-isomorphism of Sullivan $r$-minimal P-algebras. Then $f$ is an isomorphism.

Proof: By Lemma 5.1 we have a morphism $g: \mathcal{M}^{\prime} \rightarrow \mathcal{M}$ such that $f g=$ $\operatorname{id}_{\mathcal{M}^{\prime}}$. By the two out of three property, $g$ is also a quasi-isomorphism. Again, by Lemma 5.1 we have a morphism $g^{\prime}: \mathcal{M} \rightarrow \mathcal{M}$ such that $g g^{\prime}=\mathrm{id}_{\mathcal{M}}$. Therefore $g$ is both injective and surjective and hence an isomorphism and we have $f=g^{-1}$.

The main result of this section is the following:

Theorem 5.3. Let $P$ be an $r$-tame operad and let $A$ be an $r$-connected P-algebra. Let $f: \mathcal{M} \rightarrow A$ and $f^{\prime}: \mathcal{M}^{\prime} \rightarrow A$ be two Sullivan $r$-minimal models of $A$. Then there is an isomorphism $g: \mathcal{M} \rightarrow \mathcal{M}^{\prime}$, unique up to homotopy, such that $f^{\prime} g \simeq f$.

Proof: By Proposition 3.11 we obtain $g$, uniquely defined up to homotopy, such that $f^{\prime} g \simeq f$. By Lemma $5.2, g$ is an isomorphism.

Let $\operatorname{Ho}\left(\mathbf{A l g}_{P}^{r}\right)$ denote the localized category of $r$-connected $P$-algebras with respect to the class of quasi-isomorphisms. Denote by $\mathbf{S M i n}_{P}^{r} / \simeq$ the category of $r$-connected Sullivan minimal $P$-algebras, whose morphisms are homotopy classes of morphisms of $P$-algebras. We have: 
Corollary 5.4. Let $P$ be an r-tame operad. The category $\mathbf{A l g}_{P}^{r}$ of $r$-connected $P$-algebras is a Sullivan category in the sense of [GNPR]. In particular, the inclusion of minimal algebras induces an equivalence of categories

$$
\operatorname{SMin}_{P}^{r} / \simeq \stackrel{\sim}{\longrightarrow} \operatorname{Ho}\left(\operatorname{Alg}_{P}^{r}\right)
$$

Proof: For every $P$-algebra $A$, the choice of a minimal model $\mathcal{M}$ gives a well-defined functor $A \mapsto \mathcal{M}$ between the homotopy categories, which is the quasi-inverse of the functor induced by the inclusion.

\section{Algebras over variable operads}

Let $P$ be an operad and $A$ a $P$-algebra. Given two minimal models $F: P_{\infty} \rightarrow P$ and $F^{\prime}: P_{\infty}^{\prime} \rightarrow P$, we may consider the reciprocal images $F^{*}(A)$ and $F^{*}(A)$ of $A$ in the categories of $P_{\infty}$-algebras and $P_{\infty}^{\prime}$-algebras respectively. In this section, we compare the minimal models of these reciprocal images. This problem is better understood in the fibred category of algebras over all operads, which we next introduce.

Definition 6.1. Denote by Alg the category whose objects are pairs $(P, A)$ with $P \in \mathbf{O p}$ and $A \in \mathbf{A} \mathbf{l g}_{P}$ and whose morphisms $(F, f)$ : $(P, A) \rightarrow(Q, B)$ are given by a morphism $F: P \rightarrow Q$ of operads, together with a morphism $f: A \rightarrow F^{*}(B)$ of $P$-algebras. The composition of morphisms $(F, f):(P, A) \rightarrow(Q, B)$ and $(G, g):(Q, B) \rightarrow(R, C)$ is defined by $(G, g) \circ(F, f):=\left(G \circ F, F^{*}(g) \circ f\right)$. Objects in Alg will be called algebras (over variable operads).

Following the main theorem of [Roi2] and taking into account the remarks of $[\mathbf{S t a}]$, one can produce a Quillen model category structure on $\mathbf{A l g}$, from the ones on $\mathbf{O p}$ and $\mathbf{A} \mathbf{l g}_{P}$ (see $[\mathbf{B M}]$, [Hin1]). However, since here we are only interested in minimal models, we don't need the whole power of a Quillen model structure. As we have seen, in order to talk about and prove existence of minimal models it suffices to consider weak equivalences (quasi-isomorphisms). If, on top, we want to study uniqueness, we also need a notion of homotopy.

Definition 6.2. A morphism $(F, f):(P, A) \rightarrow(Q, B)$ in $\mathbf{A l g}$ is said to be a quasi-isomorphism if $F: P \rightarrow Q$ is a quasi-isomorphism of operads and $f: A \rightarrow F^{*}(B)$ is a quasi-isomorphism of $P$-algebras.

Using the notion of principal extension of an operad [MSS, Definition 3.138], we define Sullivan operads as done with operad algebras:

Definition 6.3. A Sullivan operad is the colimit of a sequence of principal extensions of arities $>1$, starting from 0 . 
Definition 6.4. We will say that a pair $(R, C) \in \mathbf{A l g}$ is a Sullivan algebra if $R$ is a Sullivan operad and $C$ is a Sullivan $R$-algebra.

Proposition 6.5. Let $(R, C)$ be a Sullivan algebra. Then for every solid diagram in $\mathbf{A l g}$

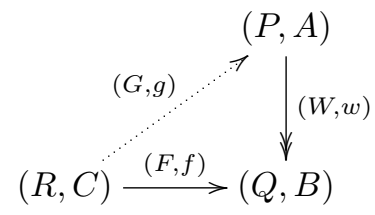

where $(W, w)$ is a surjective quasi-isomorphism, there exists $(G, g)$ making the diagram commute.

Proof: Since $R$ is a Sullivan operad, by Lemma 3.139 of [MSS] there exists a morphism $G: R \rightarrow P$ such that $W \circ G=F$. Consider the solid diagram of $P$-algebras

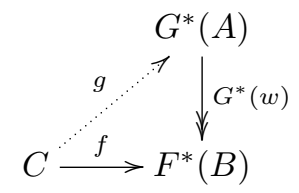

Note that since $G^{*} W^{*}=F^{*}$, this is well-defined. Since $C$ is a Sullivan $R$-algebra and $G^{*}(w)$ is a surjective quasi-isomorphism, by Proposition 3.6 there is a morphism $g$ making the diagram commute.

Definition 6.6. A functorial path in the category $\mathbf{A l g}$ is defined as the functor

$$
-[t, d t]: \mathbf{A l g} \longrightarrow \mathbf{A l g}
$$

given on objects by $(P, A)[t, d t]=(P[t, d t], A[t, d t])$ and on morphisms by $(F, f)[t, d t]=(F[t, d t], f[t, d t])$, together with the natural transformations

$$
(P, A) \stackrel{(I, \iota)}{\longrightarrow}(P[t, d t], A[t, d t]) \underset{\left(\Delta^{0}, \delta^{0}\right)}{\stackrel{\left(\Delta^{1}, \delta^{1}\right)}{\longrightarrow}}(P, A) ; \quad\left(\Delta^{k}, \delta^{k}\right) \circ(I, \iota)=\operatorname{id}_{(P, A)} .
$$

Note that if $F: P \rightarrow Q$ is a morphism of operads then $F[t, d t]^{*}=$ $F^{*}[t, d t]$.

The path gives a natural notion of homotopy between morphisms in Alg. As in Section 3, the following are classical consequences of Proposition 6.5. 
Proposition 6.7. The homotopy relation between morphisms in $\mathbf{A l g}$ is an equivalence relation for those morphisms whose source is a Sullivan algebra.

Proof: The proof follows verbatim the proof of Proposition 3.10, using Proposition 6.5.

Denote by $[(P, A),(Q, B)]$ the set of homotopy classes of morphisms of algebras from $(P, A)$ to $(Q, B)$.

Proposition 6.8. Let $(R, C)$ be a Sullivan algebra. Any quasi-isomorphism $(W, w):(P, A) \rightarrow(Q, B)$ induces a bijection $(W, w)_{*}:[(R, C)$, $(P, A)] \rightarrow[(R, C),(Q, B)]$.

Proof: The proof follows verbatim the proof of Proposition 3.11.

We now study the existence and uniqueness of minimal models in Alg.

Definition 6.9. We will say that $\left(P_{\infty}, \mathcal{M}\right) \in \mathbf{A l g}$ is a Sullivan $r$-minimal algebra if $P_{\infty}$ is a minimal operad which is $r$-tame and $\mathcal{M}$ is a Sullivan $r$-minimal $P_{\infty}$-algebra. A Sullivan $r$-minimal model for a pair $(P, A)$ is a Sullivan $r$-minimal algebra $\left(P_{\infty}, \mathcal{M}\right)$ together with a quasiisomorphism $\left(P_{\infty}, \mathcal{M}\right) \rightarrow(P, A)$.

Theorem 6.10. Let $P$ be a reduced $r$-tame operad and let $A$ be an $r$ connected P-algebra. Then $(P, A)$ has a Sullivan $r$-minimal model.

Proof: By Theorem 3.125 of [MSS], every reduced operad $P \in \mathbf{O p}$ with $H(P)(1)=\mathbf{k}$ has a minimal model $F: P_{\infty} \rightarrow P$. Since $P(1)=$ $\mathbf{k}$ this hypothesis is clearly satisfied. Furthermore, $P_{\infty}$ is $r$-tame by Proposition 4.10. Since $F^{*}(A)$ is an $r$-connected $P_{\infty}$-algebra, by Theorem 4.6 there is a Sullivan minimal $P_{\infty}$-algebra $\mathcal{M}$ together with a quasiisomorphism $f: \mathcal{M} \rightarrow F^{*}(A)$. The morphism $(F, f):\left(P_{\infty}, \mathcal{M}\right) \rightarrow(P, A)$ is a Sullivan $r$-minimal model of $(P, A)$.

Lemma 6.11. Let $(F, f):\left(P_{\infty}, \mathcal{M}\right) \rightarrow\left(P_{\infty}^{\prime}, \mathcal{M}^{\prime}\right)$ be a quasi-isomorphism of Sullivan r-minimal algebras. Then $(F, f)$ is an isomorphism.

Proof: Since $F: P_{\infty} \rightarrow P_{\infty}^{\prime}$ is a quasi-isomorphism of minimal operads, it is an isomorphism (see Theorem 3.119. of $[\mathbf{M S S}]$ ). Therefore $F^{*}$ preserves Sullivan minimal algebras and hence $f: \mathcal{M} \rightarrow F^{*} \mathcal{M}^{\prime}$ is also an isomorphism.

Remark 6.12. Note that Proposition 6.8 together with Lemma 6.11 make Sullivan minimal algebras in Alg, minimal in an abstract categorical sense (c.f. [Roi3], [Roi1], [GNPR]). 
Theorem 6.13. Let $A$ be an $r$-connected $P$-algebra. Let

$$
(F, f):\left(P_{\infty}, \mathcal{M}\right) \longrightarrow(P, A) \quad \text { and } \quad\left(F^{\prime}, f^{\prime}\right):\left(P_{\infty}^{\prime}, \mathcal{M}^{\prime}\right) \longrightarrow(P, A)
$$

be two Sullivan $r$-minimal models of $(P, A)$. Then there is an isomorphism

$$
(G, g):\left(P_{\infty}, \mathcal{M}\right) \longrightarrow\left(P_{\infty}^{\prime}, \mathcal{M}^{\prime}\right)
$$

unique up to homotopy, such that $\left(F^{\prime}, f^{\prime}\right) \circ(G, g) \simeq(F, f)$.

Proof: By Proposition 6.8 we obtain $(G, g)$, uniquely defined up to homotopy, such that $(F, f) \circ(G, g) \simeq\left(F^{\prime}, f^{\prime}\right)$. By Lemma $5.2,(G, g)$ is an isomorphism.

Denote by $\mathbf{A l g}^{r}$ the category whose objects are pairs $(P, A)$ where $P$ is a reduced $r$-tame operad and $A$ is an $r$-connected $P$-algebra and by $\mathrm{Ho}\left(\mathbf{A l g}^{r}\right)$ the localized category with respect to quasi-isomorphisms. Also, let $\mathbf{S M i n} r / \simeq$ denote the category of Sullivan $r$-minimal algebras, whose morphisms are homotopy classes of morphisms in Alg. We have:

Corollary 6.14. The category $\mathbf{A l g}^{r}$ is a Sullivan category in the sense of [GNPR]. In particular, the inclusion of minimal algebras induces an equivalence of categories

$$
\operatorname{SMin}^{r} / \simeq \stackrel{\sim}{\longrightarrow} \operatorname{Ho}\left(\mathbf{A l g}^{r}\right) .
$$

\section{Chain operad algebras and one example}

In this section, we verify that our results are also valid for chain operad algebras, i.e., algebras over operads in the category of chain complexes of $\mathbf{k}$-vector spaces (with homological grading).

Note that the proofs of Sections 3, 5, and 6 don't depend on any specific behavior of the degree of differentials. In particular, all statements and proofs admit automatic translations to the chain setting just by replacing the word cochain by the word chain everywhere in the text, together with the following minor changes:

(1) In the Definition 3.2 of a KS-extension of a free $P$-algebra $A$ by a graded vector space $V^{\prime}$ of degree $n$, the linear map is $d: V^{\prime} \rightarrow$ $Z_{n-1}(A)$ (instead of $Z^{n+1}$ ).

(2) The cone of a morphism $f: A \rightarrow B$ is in the chain setting given by $C(f)_{n}=A_{n-1} \oplus B_{n}$ with $d(a, b)=(-d a, d b-f(a))$.

(3) In the definition of the algebra $\mathbf{k}[t, d t], d t$ has degree -1 .

We next revise the construction of Sullivan minimal models of Section 4. Let us remark that in the chain setting, we keep the same definition of $r$-tame operad as in Definition 4.1. We also keep the same 
definition of Sullivan minimal $P$-algebra as a colimit of a sequence of KS-extensions ordered by non-decreasing degrees. Note that the key Lemmas 4.4 and 4.5 are still valid in the chain setting, since neither the statements nor the proofs involve any differentials.

Theorem 7.1. Let $P$ be an $r$-tame operad in chain complexes (with homological degree). Then every $r$-connected $P$-algebra $A$ has a Sullivan $r$-minimal model $f: \mathcal{M} \rightarrow A$ with $\mathcal{M}_{0}=P(0)$ and $\mathcal{M}_{i}=0$ for all $i<r$ with $i \neq 0$. Furthermore, if $A$ is $(r+1)$-connected and $H_{*}(A)$ is of finite type, then $\mathcal{M}$ is of finite type.

Proof: The proof is analogous to that of Theorem 4.6 with minor changes, as done by Neisendorfer in $[\mathbf{N e i}]$ in the case of chain Lie algebras. Let $\mathcal{M}[0]=H_{0}(A)$ and define $f_{0}: \mathcal{M}[0] \rightarrow A$ by taking a section of the projection $Z_{0}(A) \rightarrow H_{0}(A)$. Then $H_{i} f_{0}$ is trivially an isomorphism for $i<0$ and an epimorphism for $i=0$.

Assuming we have constructed $f_{n-1}: \mathcal{M}[n-1] \rightarrow A$ with $\mathcal{M}[n-$ 1] a Sullivan minimal $P$-algebra generated in degrees $<n$ and $f_{n-1}$ a morphism such that $H_{i} f_{n-1}$ is an isomorphism for $i<n-1$ and an epimorphism for $i=n-1$, we build $\mathcal{M}[n]$ in two steps:

$\left(1_{n}\right)$ The map $f_{n}^{\prime}: \mathcal{M}[n]^{\prime} \rightarrow A$ is obtained from $f_{n-1}: \mathcal{M}[n-1] \rightarrow$ $A$ after killing the kernel of $f_{n-1}$ in degree $n$. This is done by successively attaching KS-extensions of degree $n-1$ (in the $(r+1)$ connected case, only one KS-extension is needed).

$\left(2_{n}\right)$ The map $f_{n}: \mathcal{M}[n] \rightarrow A$ is obtained from $f_{n}^{\prime}: \mathcal{M}[n]^{\prime} \rightarrow A$ after killing the cokernel of $f_{n}^{\prime}$ in dimension $n+1$. This is done by a attaching KS-extension of degree $n+1$ with trivial differential.

Now, the resulting Sullivan $P$-algebra $\mathcal{M}=\cup_{n} \mathcal{M}[n]$ is not minimal, since KS-extensions are not ordered by degree. We next show that steps $\left(2_{n}\right)$ and $\left(1_{n+1}\right)$ can be permuted. Consider the sequence

$$
\begin{aligned}
\cdots \rightarrow \mathcal{M}[n]^{\prime} \longrightarrow \mathcal{M}[n]=\mathcal{M}[n]^{\prime} \sqcup_{0} P\left\langle U_{n+1}\right\rangle & \longrightarrow \mathcal{M}[n+1]^{\prime} \\
& =\mathcal{M}[n] \sqcup_{d} P\left\langle V_{n}\right\rangle \longrightarrow \cdots .
\end{aligned}
$$

Since the differential on $U_{n+1}$ is trivial, it suffices to show that

$$
d: V_{n} \longrightarrow Z_{n-1}\left(\mathcal{M}[n-1] \backslash P\left\langle U_{n+1}\right\rangle\right) \text {. }
$$

This is a direct consequence of the fact that $\mathcal{M}[n+1]_{n-1}=\mathcal{M}[n]_{n-1}^{\prime}$, by Lemma 4.5 .

To end the paper, we compute the minimal model of the chains of some double loop spaces $C_{*} \Omega^{2} X$ as $\mathcal{G}_{\infty} r_{\infty}$-algebras. We will use the the following result, valid for either the chain or cochain setting: 
Proposition 7.2. Let $P$ be an operad with zero differential, $P_{\infty}$ its minimal model, $A$ a $P_{\infty}$-algebra such that $H A=P\langle V\rangle$ is a free $P$-algebra, also with zero differential. Then $P_{\infty}\langle V\rangle$ is the minimal model of $A$ as a $P_{\infty}$-algebra.

Proof: We have a k-linear lifting $s$,

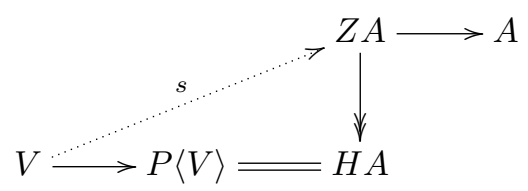

Composed with the natural inclusion $Z A \hookrightarrow A, s$ will induce a morphism of $P_{\infty}$-algebras $\rho: P_{\infty}\langle V\rangle \rightarrow A$, which is a quasi-isomorphism, since

$$
\begin{aligned}
H\left(P_{\infty}\langle V\rangle\right) & =H\left(\bigoplus_{n \geq 0} P_{\infty}(n) \otimes_{\Sigma_{n}} V^{\otimes n}\right)=\bigoplus_{n \geq 0}\left(H P_{\infty}(n) \otimes_{\Sigma_{n}} H V^{\otimes n}\right) \\
& =\bigoplus_{n \geq 0}\left(P(n) \otimes_{\Sigma_{n}} V^{\otimes n}\right)=P\langle V\rangle=H A
\end{aligned}
$$

Example 7.3. For every connected pointed topological space $X$ its double loop space $\Omega^{2} X$ has an action of the little disk operad $\mathcal{D}_{2},[\mathbf{B V}]$. Hence, the singular chain complex $C_{*} \Omega^{2} X$ is an algebra over the operad of the chain complex $C_{*} \mathcal{D}_{2}$, and the homology $H_{*} \Omega^{2} X$ is an algebra over the operad $H_{*} \mathcal{D}_{2}$. This is true with any coefficients, in particular over $\mathbb{Q}$. From now on, we assume rational coefficients everywhere.

By the results of Cohen's thesis $[\mathbf{C L M}]$, the homology $H_{*} \mathcal{D}_{2}$ is isomorphic to the Gerstenhaber operad: $H_{*} \mathcal{D}_{2} \cong G_{\mathcal{L}}$ er. Therefore every homology $H_{*} \Omega^{2} X$ carries a natural structure of a Ger-algebra. Furthermore, Tamarkin [Tam] showed that $C_{*} \mathcal{D}_{2}$ is a formal operad; that is, its minimal model in the sense of Markl is also the minimal model of its homology. This gives quasi-isomorphisms

$$
\text { Ger } \cong H_{*} \mathcal{D}_{2} \stackrel{\sim}{\sim} \mathcal{G}_{\infty} \stackrel{\sim}{\sim} C_{*} \mathcal{D}_{2}
$$

In particular, we have that every $C_{*} \mathcal{D}_{2}$-algebra is naturally a $\mathcal{G}_{2} \boldsymbol{c}_{\infty}$-algebra. More specifically, $C_{*} \Omega^{2} X$ has a natural structure of a $\mathcal{G}_{\infty} r_{\infty}$-algebra. We will compute the minimal model of some of these $C_{*} \Omega^{2} X$ as such.

For this, we rely on the fact that the rational homology $H_{*}\left(\Omega^{2} \Sigma^{2} X\right)$ of the double loop space of the double suspension of $X$ is free as a Gerstenhaber algebra, over the reduced homology of $X$ (see [Get, Section 1], cf. [GJ, Theorem 6.1], and the generalization in [SW, Theorem 6.5]):

$$
H_{*}\left(\Omega^{2} \Sigma^{2} X\right) \cong \operatorname{Ger}\left\langle\widetilde{H}_{*} X\right\rangle .
$$


By Proposition 7.2, we obtain a Sullivan minimal model as a $\mathcal{C}_{\infty}{ }_{\infty}^{- \text {al- }}$ gebra

$$
\rho: \mathcal{G}_{\infty}\left\langle\widetilde{H}_{*} X\right\rangle \stackrel{\sim}{\longrightarrow} C_{*}\left(\Omega^{2} \Sigma^{2} X\right) .
$$

For instance, for $n>2$, the minimal model of $\Omega^{2} S^{n+1}=\Omega^{2} \Sigma^{2} S^{n-1}$ is the free $\mathcal{G}_{\infty} r_{\infty}$-algebra $\mathcal{L}_{e r}\left\langle e_{n-1}\right\rangle$ on a single generator $e_{n-1}$ in degree $n-1$. See [Gin, Theorem 3.6], for a handy description of $\mathcal{G}_{\mathcal{L}} \mathrm{r}_{\infty}$-algebras.

This answers, we believe, a question of Getzler-Jones [GJ, Section 6] about Sullivan minimal models for double loop spaces being unable to reflect the Gerstenhaber structure.

Acknowledgements. The first named author would like to thank Muriel Livernet and Dennis Sullivan for helpful comments. The second named author thanks Vicenç Navarro, Pere Pascual, and Francisco Guillén for useful exchange of ideas, Neil Strickland for explaining the isomorphism describing the homology $H_{*}\left(\Omega^{2} \Sigma^{2} X\right)$ as a free Gerstenhaber algebra, and Andy Tonks for providing valuable references. We would also like to thank Daniel Tanré for his useful questions as well as the referees for their questions and suggestions.

\section{References}

[BL] H. J. Baues and J. M. Lemaire, Minimal models in homotopy theory, Math. Ann. 225(3) (1977), 219-242. DOI : 10.1007/BF01425239.

[BM] C. Berger and I. MoerdiJK, Axiomatic homotopy theory for operads, Comment. Math. Helv. 78(4) (2003), 805-831. DOI : 10.1007/s00014-0030772-y.

[BV] J. M. Boardman and R. M. Vogt, "Homotopy Invariant Algebraic Structures on Topological Spaces", Lecture Notes in Mathematics 347, SpringerVerlag, Berlin-New York, 1973. DOI : 10.1007/BFb0068547.

[CL] J. ChuAng and A. Lazarev, Feynman diagrams and minimal models for operadic algebras, J. Lond. Math. Soc. (2) 81(2) (2010), 317-337. DOI: $10.1112 / \mathrm{j} 1 \mathrm{~ms} / \mathrm{jdp} 073$.

[Cir] J. Cirici, Cofibrant models of diagrams: Mixed Hodge structures in rational homotopy, Trans. Amer. Math. Soc. 367(8) (2015), 5935-5970. DOI: 10.1090/S0002-9947-2014-06405-2.

[CLM] F. R. Cohen, T. J. LAda, And J. P. MaY, "The Homology of Iterated Loop Spaces", Lecture Notes in Mathematics 533, Springer-Verlag, Berlin-New York, 1976. DOI : 10.1007/BFb0080464.

[FHT] Y. Félix, S. Halperin, And J.-C. Thomas, "Rational Homotopy Theory", Graduate Texts in Mathematics 205, Springer-Verlag, New York, 2001. DOI : $10.1007 / 978-1-4613-0105-9$.

[Fre] B. Fresse, "Modules over Operads and Functors", Lecture Notes in Mathematics 1967, Springer-Verlag, Berlin, 2009. DOI : 10.1007/978-3-54089056-0.

[GeMa] S. I. Gelfand And Yuri I. Manin, "Methods of Homological Algebra", Second edition, Springer Monographs in Mathematics, Springer-Verlag, Berlin, 2003. DOI : $10.1007 / 978-3-662-12492-5$. 
[Get] E. Getzler, Batalin-Vilkovisky algebras and two-dimensional topological field theories, Comm. Math. Phys. 159(2) (1994), 265-285. DOI: 10.1007/BF02102639.

[GJ] E. Getzler and J. D. S. Jones, Operads, homotopy algebra and iterated integrals for double loop spaces, Preprint (1994). arXiv:hep-th/9403055.

[Gin] G. Ginot, Homologie et modèle minimal des algèbres de Gerstenhaber, Ann. Math. Blaise Pascal 11(1) (2004), 95-126. DOI: 10.5802/ambp.187.

[Góm] A. Gómez Tato, Modèles minimaux résolubles, J. Pure Appl. Algebra 85(1) (1993), 43-56. DOI : 10.1016/0022-4049(93) 90170-X.

[GrMo] P. Griffiths AND J. Morgan, "Rational Homotopy Theory and Differential Forms", Second edition, Progress in Mathematics 16, Springer, New York, 2013. DOI : $10.1007 / 978-1-4614-8468-4$.

[GNPR] F. Guillén, V. Navarro, P. Pascual, and A. Roig, A Cartan-Eilenberg approach to homotopical algebra, J. Pure Appl. Algebra 214(2) (2010), 140-164. DOI : $10.1016 / j$.jpaa.2009.04.009.

[Hin1] V. Hinich, Homological algebra of homotopy algebras, Comm. Algebra 25(10) (1997), 3291-3323. DOI : 10.1080/00927879708826055.

[Hin2] V. HiNICH, Virtual operad algebras and realization of homotopy types, $J$. Pure Appl. Algebra 159(2-3) (2001), 173-185. DOI : 10.1016/S0022-4049 (00) 00054-2.

[Kad] T. V. KADEIŠVILI, On the homology theory of fibre spaces, (Russian), International Topology Conference (Moscow State Univ., Moscow, 1979), Uspekhi Mat. Nauk 35 (1980), no. 3(213), 183-188; translation in: Russian Mathematical Surveys 35(3) (1980), 231-238. DOI : 10.1070/RM1980v035n03ABEH 001842.

[KP] K. H. Kamps And T. Porter, "Abstract Homotopy and Simple Homotopy Theory", World Scientific Publishing Co., Inc., River Edge, NJ, 1997. DOI : 10.1142/9789812831989.

[KM] I. Kříž AND J. P. MAY, Operads, algebras, modules and motives, Astérisque 233 (1995), 145 pp.

[Liv1] M. Livernet, Homotopie rationnelle des algèbres sur une opérade, Thèse, Université Louis Pasteur (Strasbourg I) (1998).

[Liv2] M. Livernet, Rational homotopy of Leibniz algebras, Manuscripta Math. 96(3) (1998), 295-315. DOI : 10.1007/s002290050069.

[Lod] J.-L. LODAY, The diagonal of the Stasheff polytope, in: "Higher Structures in Geometry and Physics", Progr. Math. 287, Birkhäuser/Springer, New York, 2011, pp. 269-292. DOI : 10.1007/978-0-8176-4735-3-13.

[LV] J.-L. Loday and B. Vallette, "Algebraic Operads", Grundlehren der Mathematischen Wissenschaften 346, Springer, Heidelberg, 2012. DOI: 10.1007/978-3-642-30362-3.

[MS] M. Markl AND S. ShNider, Associahedra, cellular $W$-construction and products of $A_{\infty}$-algebras, Trans. Amer. Math. Soc. 358(6) (2006), 2353-2372. DOI : $10.1090 /$ S0002-9947-05-04006-7.

[MSS] M. Markl, S. Shnider, and J. StashefF, "Operads in Algebra, Topology and Physics", Mathematical Surveys and Monographs 96, American Mathematical Society, Providence, RI, 2002. DOI : 10.1090/surv/096.

[Mil1] J. Millès, André-Quillen cohomology of algebras over an operad, Adv. Math. 226(6) (2011), 5120-5164. DOI : 10.1016/j.aim.2011.01.002. 
[Mil2] J. MilLÈs, The Koszul complex is the cotangent complex, Int. Math. Res. Not. IMRN 2012(3) (2012), 607-650. DOI : 10.1093/imrn/rnr034.

[Nei] J. A. Neisendorfer, Lie algebras, coalgebras and rational homotopy theory for nilpotent spaces, Pacific J. Math. 74(2) (1978), 429-460. DOI: 10.2140/pjm.1978.74.429.

[Qui] D. QuilLEn, Rational homotopy theory, Ann. of Math. (2) 90(2) (1969), 205-295. DOI : $10.2307 / 1970725$.

[Roi1] A. Roig, Minimal resolutions and other minimal models, Publ. Mat. 37(2) (1993), 285-303. DOI : 10.5565/PUBLMAT_37293_04.

[Roi2] A. Rolg, Model category structures in bifibred categories, J. Pure Appl. Algebra 95(2) (1994), 203-223. DOI : 10.1016/0022-4049(94)90074-4.

[Roi3] A. Roig, Modèles minimaux et foncteurs dérivés, J. Pure Appl. Algebra 91(1-3) (1994), 231-254. DOI : 10.1016/0022-4049(94)90145-7.

[SW] P. Salvatore And N. Wahl, Framed discs operads and Batalin-Vilkovisky algebras, Q. J. Math. 54(2) (2003), 213-231. DOI: 10.1093/qjmath/54.2. 213.

[SU] S. Saneblidze and R. Umble, Diagonals on the permutahedra, multiplihedra and associahedra, Homology Homotopy Appl. 6(1) (2004), 363-411. DOI : 10.4310/HHA. 2004.v6.n1.a20.

[Sta] A. E. Stanculescu, Bifibrations and weak factorisation systems, Appl. Categ. Structures 20(1) (2012), 19-30. DOI : 10.1007/s10485-009-9214-3.

[Sul1] D. Sullivan, Infinitesimal computations in topology, Inst. Hautes Études Sci. Publ. Math. 47(1) (1977), 269-331 (1978). DOI : 10.1007/BF02684341.

[Sul2] D. Sullivan, Homotopy theory of the master equation package applied to algebra and geometry: a sketch of two interlocking programs, in: "Algebraic Topology-Old and New", Banach Center Publ. 85, Polish Acad. Sci. Inst. Math., Warsaw, 2009, pp. 297-305. DOI : 10.4064/bc85-0-20.

[Tam] D. E. Tamarkin, Formality of chain operad of little discs, Lett. Math. Phys. 66(1-2) (2003), 65-72. DOI : 10.1023/B:MATH.0000017651.12703.a1.

Joana Cirici

Fachbereich Mathematik und Informatik, Universität Münster, Einsteinstrasse 62, 48149 Münster, Germany

E-mail address: jcirici@gmail.com

Agustí Roig

Departament de Matemàtiques, Universitat Politècnica de Catalunya, UPC, Diagonal 647, 08028 Barcelona, Spain

E-mail address: agustin.roig@upc.edu

Primera versió rebuda el 13 de març de 2017, darrera versió rebuda el 8 de gener de 2018. 